\title{
Diversité des techniques et pratiques culturales du fonio (Digitaria exilis [Kippist] Stapf) en Afrique de l'Ouest (synthèse bibliographique)
}

\author{
Cyrille Kanlindogbe ${ }^{(1)}$, Emmanuel Sekloka ${ }^{(1)}$, Valérien Amégnikin Zinsou ${ }^{(1)}$, \\ Armand Natta ${ }^{(2)}$ \\ (1) Université de Parakou. Faculté d'Agronomie, Laboratoire de Phytotechnie, d'Amélioration et de Protection des Plantes. \\ BP 123. Parakou (Bénin).E-mail : cyrillekanlindogbe@yahoo.fr \\ (2) Université de Parakou. Faculté d'Agronomie, Laboratoire d'Écologie, de Botanique et de Biologie végétale. BP 123. \\ Parakou (Bénin).
}

Reçu le 12 avril 2019, accepté le 26 mai 2020, mis en ligne le 2 juillet 2020.

Cet article est distribué suivant les termes et les conditions de la licence CC-BY (http://creativecommons.org/licenses/by/4.0/ deed.fr)

Introduction. À la différence des grandes cultures, le fonio, une céréale négligée, n'a pu bénéficier d'un grand nombre de recherches formalisées sur les techniques culturales. Sur la base d'une recherche documentaire systématisée, cet article présente une analyse critique des techniques pratiquées en culture du fonio en Afrique subsaharienne.

Littérature. Le fonio est cultivé au regard de ses potentiels alimentaire, socioculturel, thérapeutique, économique voire fourrager. Peu exigeant, il se développe dans une large gamme de conditions pédoclimatiques avec une préférence pour les sols légers et riches en matières organiques, et les zones à pluviométrie annuelle moyenne de 900-1000 mm. Dans les pays producteurs, les variétés et les techniques culturales sont demeurées traditionnelles. Les semis se font principalement à la volée. Contrairement aux autres céréales, le fonio se cultive assez souvent sans fumure minérale ni pesticide chimique et apparait comme une culture biologique valorisable en agroécologie. Les rendements sont généralement faibles et souvent inférieurs à $1 \mathrm{t} \cdot h \mathrm{~h}^{-1}$. Ceci est surtout lié à l'absence de variétés améliorées et de pratiques culturales appropriées, au phénomène de verse, à l'égrenage spontané et au défaut de remplissage des grains.

Conclusions. Les pratiques culturales du fonio, restées traditionnelles, sont caractérisées par la pénibilité des opérations et la faiblesse des rendements. Des recherches innovantes sur les variétés, les techniques culturales et sur la mécanisation et la rentabilité permettront de lever les contraintes et de favoriser une relance effective de la production.

Mots-clés. Digitaria exilis, technique de culture, mécanisation, rendement des cultures, agroécologie, Afrique subsaharienne.

Diversity of techniques and cultivation practices of fonio millet (Digitaria exilis [Kippist] Stapf) in West Africa. A review Introduction. Unlike field crops, fonio, known to be a neglected plant, has not benefited sufficiently from formalized research on crop techniques. Based on systematic documentary research, this article presents a critical analysis of techniques practiced in fonio cultivation in Sub-Saharan Africa.

Literature. Fonio is cultivated not only as a foodstuff, but also for its potential socio-cultural, therapeutic, and economic benefits, and even for use as forage. The cereal develops in a wide range of soil and climate conditions, with a preference for light soils that are rich in organic matter, and areas with an average annual rainfall of 900-1,000 $\mathrm{mm}$. In producing countries, fonio varieties and cultivation techniques have remained traditional. Sowing is done mainly by broadcasting. Unlike other staple cereals, fonio is quite often grown without the application of chemical fertilizers or pesticides, and it appears to be a valuable biologic crop in agroecology. Yields are generally low and often less than $1 \mathrm{t} \cdot \mathrm{ha}^{-1}$. This is mainly due to the absence of improved varieties and appropriate crop practices, and to lodging phenomena, seed shattering and lack of grain filling.

Conclusions. Fonio cultivation practices have remained traditional, characterized by difficult operations and low yields. Innovative research on varieties and cultivation techniques, and on mechanization and profitability, will remove constraints and promote an effective revival of production.

Keywords. Digitaria exilis, crop techniques, mechanization, crop yield, agroecology, Sub-Saharan Africa. 


\section{INTRODUCTION}

Les espèces alimentaires négligées et sous-utilisées jouent un rôle crucial pour la sécurité alimentaire et pour la lutte contre la pauvreté des populations humaines, notamment rurales. C'est le cas du fonio (Digitaria exilis [Kippist] Stapf), une céréale africaine qui, malgré ses potentiels alimentaire, socioculturel, thérapeutique et économique, reste mineure et peu valorisée (Barnaud \& Billot, 2011 ; Cruz et al., 2011). Le fonio est cultivé dans une région s'étendant du Sénégal au lac Tchad (Portères, 1976). Bien adapté aux conditions pédoclimatiques locales, le fonio est tolérant à la sècheresse grâce à son métabolisme en C4 et contribue à la protection écologique en garantissant une bonne couverture végétale sur des sols fragiles et peu valorisés (Vall et al., 2008 ; Cruz et al., 2011). Consommé sous diverses formes (pâtes, bouillies, couscous, etc.), le fonio est indiqué pour les enfants, les personnes âgées et celles souffrant d'obésité ainsi que pour les femmes enceintes et allaitantes, vu la meilleure digestibilité de ses protéines et glucides, comparativement à celles du sorgho et du mil (Jideani, 1990 ; Vodouhè \& Achigan Dako, 2006 ; Cruz et al., 2011). Il est de surcroît pauvre en gluten et serait indiqué pour les diabétiques en raison de son indice glycémique relativement faible (environ 66 contre 95 pour le riz) (Jideani, 1990 ; Traore et al., 2009). Il est riche en méthionine et en cystéine, deux acides aminés essentiels pour l'homme et déficients dans le blé, le riz, le maïs et le sorgho (Jideani, 1990). Malgré ses multiples vertus et potentiels, il est resté une céréale marginale et négligée dans les programmes nationaux de recherche. Cet article présente une synthèse et une analyse critique des pratiques culturales du fonio en Afrique de l'Ouest pour identifier les défis et perspectives en vue d'une relance effective de sa production.

Cette recherche bibliographique a consisté en une recherche documentaire systématisée sur l'itinéraire technique du fonio via les moteurs Google Scholar, AJOL, DOAJ, AGORA et ResearchGate. Les mots-clés de l'itinéraire technique «pratiques culturales + fonio, production + fonio, exigences agro-écologiques + fonio, systèmes de culture + fonio, préparation du sol + fonio, variétés de fonio cultivées, semis du fonio, entretiens + fonio, fertilisation + fonio, nuisibles + fonio, récolte et conservation du fonio » ont été utilisés. Cette exploration a conduit à 5083 documents de différentes natures (articles, actes de colloques, thèses, mémoires, rapports et données statistiques). Un tri sélectif basé sur le choix des documents abordant les sous-thèmes développés et priorisant les publications scientifiques a permis de réduire le nombre de documents et d'en retenir 47 dans cet article. Cette documentation a été soumise à une analyse systémique et critique.

\section{EXIGENCES AGROÉCOLOGIQUES}

Le fonio crôit principalement sous des climats tropicaux avec une extension dans la zone subéquatoriale (Froment \& Renard, 2001). Son aire de préférence est la zone soudano-guinéenne (Vodouhè et al., 2003).

Le fonio est une plante peu exigeante qui s'accommode d'une large gamme de sols (légers, sablonneux, limoneux, caillouteux, superficiels, pauvres, dégradés et lessivés) (Vodouhè et al., 2003 ; CIRAD, 2009 ; Fofana et al., 2017). Il peut se développer sur des sols à $\mathrm{pH}$ acide à forte concentration en aluminium et sur les cuirasses latéritiques pauvres ne pouvant supporter d'autres cultures. Il n'affectionne pas cependant les sols trop lourds ou argileux (Froment \& Renard, 2001 ; Vodouhè et al., 2003) ainsi que les sols salés, inondés ou hydromorphes (Vodouhè \& Achigan Dako, 2006). Au Bénin, il pousse sur les sols ferrugineux, sablo-limoneux, indurés, les flancs des montagnes jusqu'aux grandes dépressions des vallées où l'hydromorphie est temporaire (Vodouhè et al., 2003). En Guinée, le fonio se cultive en altitude à plus de 1000 m (Cruz et al., 2007 ; CIRAD, 2009).

Les gammes de conditions édaphiques mentionnées témoignent d'une grande capacité d'adaptation de la culture du fonio. Cependant, les meilleurs rendements s'obtiennent sur des sols profonds, fertiles et riches en matières organiques (Cissé, 1975).

Du point de vue pluviométrique, la production est concentrée dans des régions à pluviométrie annuelle moyenne de 900-1000 mm (Vodouhè et al., 2003 ; Vodouhè \& Achigan Dako, 2006 ; Cruz et al., 2007 ; Cruz et al., 2011). Cependant, Vodouhè \& Achigan Dako (2006) rapportent que sa culture est possible même sous des isohyètes de $150 \mathrm{~mm}$ par an, tandis que Cruz et al. (2011) indiquent qu'il peut se développer dans des zones à forte pluviosité (>1500 $\mathrm{mm}$ de pluie). La possibilité de cultiver du fonio dans ces larges fourchettes de pluviosité souligne sa forte capacité d'adaptation aux variabilités pluviométriques.

Le fonio supporte des températures élevées durant le cycle cultural avec une moyenne favorable allant de $25^{\circ} \mathrm{C}$ à $30^{\circ} \mathrm{C}$ (CIRAD, 2009; Cruz et al., 2011). La température optimale durant la germination-levée au champ est de l'ordre de $30{ }^{\circ} \mathrm{C}$ et les basses températures seraient responsables d'un raccourcissement du cycle (Portères, 1955 ; Cissé, 1975).

Par ailleurs, le fonio est photopériodique avec un optimum de 11 h 30 (Portères, 1955). De plus, la photopériode permet de caractériser des variétés de jours courts et des variétés insensibles à la longueur du jour (Aliero \& Morakinyo, 2005). 


\section{SYSTÈMES DE CULTURE DU FONIO}

Le fonio est souvent cultivé hors assolement, mais peut également entrer dans un plan de rotation culturale (CIRAD, 2009). Peu exigeant, il vient souvent en fin de rotation car, sur un sol trop riche, le ratio grain/paille est au détriment du grain et sa végétation devient trop exubérante (Cruz et al., 2007). Mieux, le fonio n'est pratiqué en tête de rotation qu'en cas de monoculture sur plusieurs cycles avec des jachères de courtes durées (Cruz et al., 2011). Au Sénégal, il est souvent cultivé après une culture d'arachide ou de sorgho et parfois après une jachère (Fall et al., 2005 ; Gueye, 2007). En Guinée, il entre couramment dans trois plans de rotation culturale suivant le degré de fertilité des sols (CRA Bareng, 2003) :

- riz - fonio - fonio - jachère ;

- fonio - arachide - fonio - jachère ;

- riz - riz - fonio - jachère - sorgho - maïs - fonio jachère.

Au Bénin, le sorgho, le mil et le riz pluvial ont été rapportés comme bons précédents culturaux du fonio (Vodouhè et al., 2003). Des tendances semblables ont été rapportées au Nigeria et au Togo où le fonio se cultive même en association avec des légumineuses (arachide, pois cajan, etc.) et parfois avec le manioc et le gombo (Kwon-Ndung et al., 2001 ; AdoukonouSagbadaja et al., 2006). Il ressort que le fonio s'insère bien dans une large gamme de systèmes de production.

Par ailleurs, en rotation culturale, le fonio contrôlerait le cycle de vie des nuisibles comme l'ont montré les travaux de Ndiaye et al. (2008). En effet, ces derniers ont révélé que deux à trois rotations niébé - fonio ont induit une réduction rapide de plus de $80 \%$ du taux d'inoculum de Macrophomina phasseolina, champignon responsable d'importantes nuisances sur le niébé en zone sahélienne.

Bien que principalement cultivé en monoculture, le fonio présente de bonnes affinités culturales avec d'autres céréales et les légumineuses. Il peut ainsi être cultivé en association avec le sorgho, le mil, le maïs ou le riz pluvial (Vodouhè et al., 2003 ; Cruz et al., 2011).

\section{VARIÉTÉS CULTIVÉES}

Les cultivars du fonio sont essentiellement des variétés locales issues de la sélection paysanne. Les critères majeurs de distinction souvent utilisés par les agriculteurs sont les couleurs des racèmes et la précocité (Adoukonou-Sagbadja et al., 2006 ; Cruz et al., 2007).

$\mathrm{Au}$ regard de la couleur des racèmes, le groupe des racèmes blanchâtres appelés localement Iporapia en ditammari et le groupe des racèmes rougeâtres appelés Iporawan (ditammari) ont été habituellement recensés au Bénin (Adoukonou-Sagbadja et al., 2006 ; Dansi et al., 2010 ; Sekloka et al., 2015). Les mêmes catégorisations ont été notées au Togo (AdoukonouSagbadja et al., 2004) et en Guinée (CRA Bareng, 2003 ; Vodouhè et al., 2003) avec un troisième groupe à péricarpe ou racème noir appelé Bhalè en peulh.

La classification en fonction de la précocité a permis de distinguer assez nettement les variétés extra-précoces ( $\leq 90$ jours) des variétés tardives $(>120$ jours). Les variétés extra-précoces ont un cycle plus court de l'ordre de 2,5 mois au Mali, Burkina Faso, Sénégal et de l'ordre de 3 mois en Guinée, au Bénin, Togo et au Nigeria (Tableau 1). Les variétés tardives sont presque partout considérées comme ayant un cycle supérieur à 4 mois. Entre ces deux extrêmes, on caractérise des variétés précoces de cycle inférieur à 110 jours au Nigeria, en Guinée et au Bénin ainsi que des variétés intermédiaires ou semi-tardives dont les durées de cycle varient suivant les pays (Tableau 1).

Les variétés précoces sont souvent semées tôt (mai) pour être récoltées tôt également (aout et septembre) durant les périodes de soudure alimentaire (Vall et al., 2011). Cette précocité permet aussi de contourner les difficultés liées au retard d'installation ou d'arrêt précoce des pluies. Quant aux variétés tardives, elles sont généralement plus productives et cultivées pour constituer la réserve alimentaire principale du ménage (Dansi et al., 2010 ; Sekloka et al., 2015)

\section{PRÉPARATION DU SOL POUR LA CULTURE DU FONIO}

Le défrichage est manuel avec ou sans incinération, suivi parfois d'un épandage des cendres sur la parcelle (Cruz et al., 2007 ; Cruz et al., 2011). Traditionnellement, la préparation du sol est limitée à un léger grattage sur quelques centimètres, voire un ameublissement superficiel (Cruz et al., 2007). Pour Fall et al. (2005), le travail du sol consiste en un pseudo-labour avec la charrue ou un grattage superficiel avec la houe ou la daba.

En cas de labour manuel, Vodouhè et al. (2003) ont recommandé d'atteindre au moins $10 \mathrm{~cm}$ de profondeur. Renoux \& Dumas (1905) ont affirmé avoir obtenu de très bons résultats pour un labour de $15-20 \mathrm{~cm}$ de profondeur. En Guinée, un labour de déchaumage de 20-25 cm est recommandé sur sol argileux (Diallo et al., 2008). Sur les grandes superficies, le labour à la charrue attelée aux animaux (bœuf, cheval) tend à se développer car il est plus rapide et moins fatigant que la technique manuelle (USAID, 2008 ; Cruz et al., 2011).

Après le labour, le passage au hersage pour ameublir et affiner le sol n'est pas généralement pratiqué avant 


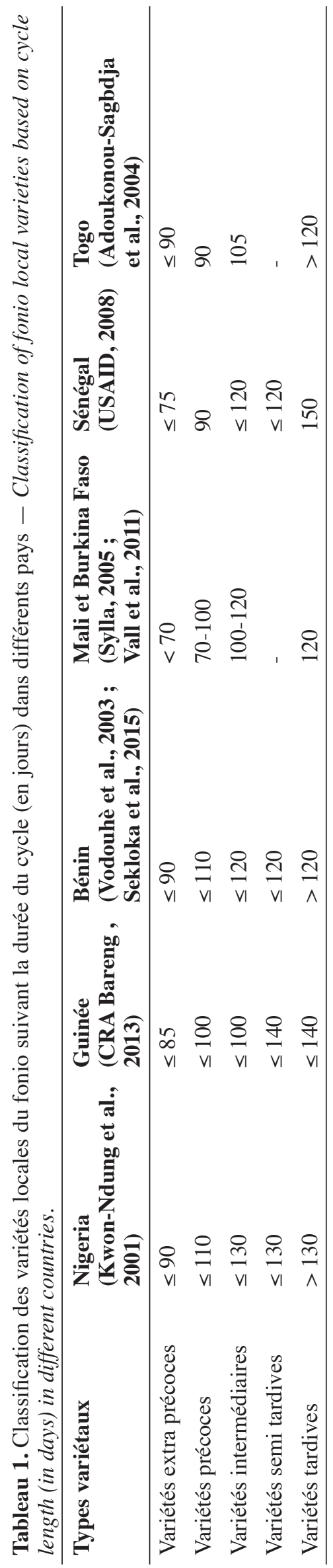

le semis en milieu réel de culture. Cependant, la recherche indique comme nécessaire l'aplanissement et l'affinement du sol pour avoir un bon lit de semis, condition favorable à une bonne germination (Fofana et al., 2017), surtout vu la petitesse des grains de fonio.

En définitive, les exigences en termes de préparation du sol ne sont pas spécifiques pour le fonio.

\section{SEMIS DU FONIO}

Le fonio se multiplie par graine. Les graines bien mûres peuvent garder leur pouvoir germinatif pendant deux campagnes, mais les semences de bonne qualité prélevées sur la dernière récolte germent mieux au bout de 2-4 jours (Portères, 1955 ; Cruz et al., 2011). En général, les semences de fonio ne sont pas traitées. Le non-traitement des semences, les conditions de leur conservation paysanne, les dégâts d'oiseaux granivores et d'insectes, les pluies violentes voire les poches de sècheresse après semis affectent énormément la levée des grains du fonio (Portères, 1955).

Des travaux de traitements à base d'insecticide fongicide avec du produit Super-Homaï 70\% WP (thiophanate-méthyl + thiram + diazinon) à raison de $250 \mathrm{~g}$ pour $10 \mathrm{~kg}$ de semences ont été rapportés pour maintenir la qualité germinative des semences (Vodouhè et al., 2003). Une étude de l'effet de différentes concentrations d'hormones sur la germination du fonio a montré des taux de germination élevés $(78 \%)$ lorsque les semences sont traitées à la thiourée (éthylène) pendant $15 \mathrm{~min}$ et $60 \mathrm{~min}$ avec des concentrations respectives de $0,01 \mathrm{mg} \cdot l^{-1}$ et de $0,0001 \mathrm{mg} \cdot \mathrm{l}^{-1}$ dans l'obscurité (Idu et al., 2008). Cissé a montré, en 1975, l'importance des hormones dans la levée de la dormance, notamment un traitement des semences à base de nitrate de potassium pouvant stimuler la germination jusqu'à $80 \%$ de levée sous la lumière. Ces différents résultats soulignent bien le rôle stimulateur des phytohormones dans la germination du fonio.

Les périodes du semis varient selon les zones agroécologiques, le cycle variétal et surtout suivant les périodes d'installation des pluies. Mais, en général, dans l'ensemble des pays producteurs, le semis du fonio s'étend du mois de mai au mois de juillet (Vodouhè et al., 2003 ; Cruz et al., 2007 ; Cruz et al., 2011). Les semis se font même parfois avant les premières pluies. Ainsi, au Mali comme au Burkina Faso, les semis se pratiquent généralement à sec dès le mois de mai ou dès la première pluie après un houage superficiel du sol (Cruz et al., 2007).

Le mode de semis du fonio le plus répandu est la volée. Les semences sont parfois mélangées à part égale du sable ou de la cendre pour favoriser une répartition homogène (Cruz et al., 2007 ; Cruz et al., 2011). La 
profondeur de semis fait aussi partie des facteurs les plus incriminés dans la mauvaise levée des graines de fonio (Portères, 1955). Ainsi, les semences du fonio, de petites tailles, sont enfouies très superficiellement, à faible profondeur, par un léger binage à la daba, un rapide hersage à la main ou en frottant des branchages d'arbres sur le sol ensemencé. Ce léger enfouissement est nécessaire également pour éviter que les graines ne soient consommées par les oiseaux granivores ou pillées par les fourmis (Portères, 1955 ; Cissé, 1975 ; Cruz et al., 2011). Parfois, un semis direct peut être réalisé sur les sols gravillonnaires enherbés suivi d'un sarclage pour l'enfouissement des grains (Diallo et al., 2008).

Portères (1955) a montré l'existence d'une profondeur de semis optimale théorique de $2 \mathrm{~cm}$ et un horizon létal à la limite de $6 \mathrm{~cm}$.

Dans la littérature, il n'existe pas un consensus sur la quantité de semences appliquée à l'hectare. Les doses de semences varient généralement en fonction de la densité attendue et du travail à consacrer à l'entretien des parcelles (USAID, 2008). Sur les parcelles labourées à la charrue, la quantité de semences utilisées peut être plus importante en considérant que certaines graines enfouies trop profondément ne vont pas bien germer (Cruz et al., 2011). Les différents travaux réalisés au Nigeria, au Sénégal ou en Guinée sur le semis à la volée ont montré que les agriculteurs utilisent des doses allant de 10 à $50 \mathrm{~kg} \cdot \mathrm{ha}^{-1}$, voire jusqu'à plus de $80 \mathrm{~kg}$. ha $^{-1}$ (Portères, 1955 ; Cissé, 1975 ; Philip \& Itodo, 2006 ; Cruz et al., 2011). Il ressort que le semis à la volée exige une grande quantité de semences et, par conséquent, un prélèvement important sur la récolte. Les fortes doses sont appliquées dans le but de limiter la compétition des adventices et accroître la réussite du semis. Cependant, il a été aussi spécifié que les fortes doses favorisent la verse des plants à maturité, tandis que les faibles doses induisent un enherbement précoce (Fofana et al., 2017). Plusieurs études ont souligné que la quantité de $30 \mathrm{~kg} \cdot \mathrm{ha}^{-1}$ constituait la dose optimale, voire la norme d'ensemencement qui améliore le rendement du fonio (Gueye, 2016 ; Fofana et al., 2017) d'environ $20 \%$ (CRA Bareng, 2003).

Par ailleurs, le semis en ligne a également été testé. Il a été établi que cette pratique réduit la quantité de semences nécessaires et facilite l'élimination des mauvaises herbes entre les lignes (Cruz et al., 2011). Réalisé en station expérimentale au moyen d'un semoir, il a nécessité seulement $15-20 \mathrm{~kg}$ de semences par hectare (Renoux \& Dumas, 1905). Cissé (1975) a mentionné également des doses de semences plus faibles de $5-10 \mathrm{~kg} \cdot \mathrm{ha}^{-1}$ en système de semis en ligne du fonio. Au Bénin, les travaux récents du semis en ligne continuent à donner des résultats similaires tout aussi intéressants en induisant une augmentation du rendement en grain du fonio de l'ordre de $150 \mathrm{~kg} \cdot \mathrm{ha}^{-1}$ à près de $200 \mathrm{~kg} \cdot \mathrm{ha}^{-1}$ avec facilitation du désherbage à la houe par rapport à la pratique de la volée (Kanlindogbe et al., 2018). En plus, un semis en lignes distantes de 25 à $30 \mathrm{~cm}$ rend les sarclages faciles et offre la possibilité de les réaliser avec un cultivateur mécanique (Cruz et al., 2011). Néanmoins, des travaux plus affinés sur les semis en lignes continues et en poquets avec combinaison de divers écartements de semis paraitraient importants afin de trouver un optimum de production, comme ce fut le cas pour d'autres céréales (riz, maïs, etc.). Cependant, le semis manuel en ligne est très laborieux et demanderait plus d'une journée de travail par hectare $\left(27-36 \mathrm{~h} \cdot \mathrm{ha}^{-1}\right)$, soit environ sept à neuf fois la durée de travail pour le semis à la volée (4h.ha-1) (Kanlindogbe et al., 2018).

Ainsi, vu le temps relativement élevé de préparations manuelles des lignes et du semis, il s'avérerait nécessaire de recourir aux outils mécaniques munis de semoirs, accessibles à moindre cout aux agriculteurs, afin de faciliter l'adoption du semis en ligne.

Une étude sur la rentabilité et l'accessibilité de la mécanisation permettrait l'adoption de cette technologie de semis en ligne en zones de culture de fonio.

\section{AMENDEMENT ET FERTILISATION DES SOLS SOUS LA CULTURE DU FONIO}

En général, le fonio se cultive sans apport de fumure minérale et bénéficie des arrières effets des antécédents culturaux lorsqu'il est inclus dans un plan de rotation (Cruz et al., 2007).

Toutefois, des efforts significatifs de recherche ont été fournis sur la fertilisation du fonio dans plusieurs pays (Tableau 2). Il est apparu que le fonio répond positivement aux apports de fumure. En termes de fumure organique, il semble se dégager une dose de 5 tha ${ }^{-1}$ (Tableau 2). Quant à la fertilisation minérale, des doses modérées de $\mathrm{N}$, $\mathrm{P}$ et $\mathrm{K}$ sont recommandées (Gigou et al., 2009) (Tableau 2). Une fertilisation de fond en matières organiques comme le fumier, les sousproduits végétaux, les engrais verts et les antécédents culturaux constitue une alternative à l'utilisation des engrais chimiques (Gigou et al., 2009 ; Cruz et al., 2011).

Par ailleurs, les travaux de Ndoye et al. (2016) ont suggéréquel'utilisation des champignons mycorhiziens à arbuscule comme biofertilisant pourrait constituer une stratégie prometteuse pour l'amélioration de la productivité du fonio au Sénégal et dans d'autres pays ouest-africains. En effet, l'inoculation réalisée à base de champignons mycorhiziens à arbuscule a induit une augmentation des paramètres de croissance et de la biomasse totale jusqu'à $45 \%$. 


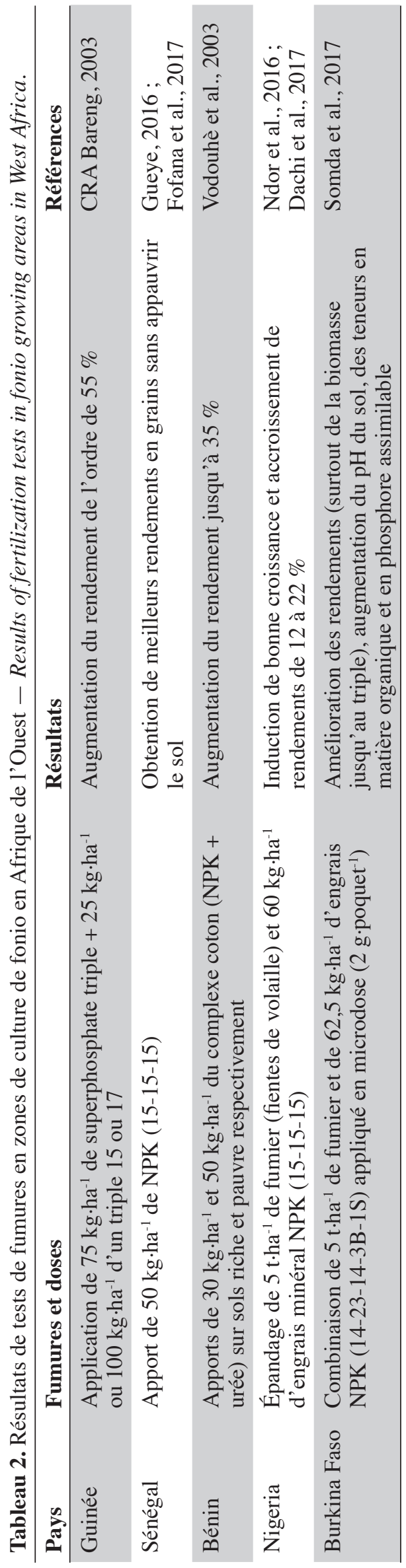

Ces différents résultats suggèrent qu'une fertilisation organo-minérale avec une bonne combinaison de doses modérées d'engrais minéral et de fumure organique ou d'autres biofertilisants améliorerait significativement les rendements du fonio (Tableau 2).

\section{MAUVAISES HERBES DU FONIO ET MESURES DE LUTTE}

L'inventaire réalisé par Ouedraogo et al. (2015) dans la zone ouest du Burkina Faso a souligné la prédominance des Poaceae et des Cyperaceae dans la flore des adventices en culture du fonio. Les espèces de mauvaises herbes les plus rapportées étaient Striga hermontica (Delile) Benth., Striga rowlandii Engl., Striga gesneroides (Willd.) Vatke, Eleusine indica (L.) Gaertn., Spermacoce ruelliae DC., Digitaria cilliaris (Retz.) Koeler et Digitaria horizontalis Willd (Haq \& Ogbe, 1995 ; Vodouhè et al., 2003 ; Gueye, 2016). Parmi elles, S. gesneroides et D.horizontalis ont été mentionnées comme les plus redoutables parasites à fortes concurrences nutritive, hydrique et lumineuse (Bakare, 2005 ; Ouedraogo et al., 2015).

Les moyens de lutte se résument fréquemment à des opérations de désherbages manuels qui semblent constituer le principal goulot d'étranglement. Dans l'ensemble des pays producteurs, l'entretien varie d'un à trois désherbages/sarclages. En Guinée, au Sénégal et au Nigeria, deux désherbages manuels ou à la houe sont effectués respectivement aux environs de 30 et 50 jours après semis. En cas d'enherbement excessif, un troisième désherbage peut être envisagé (Cruz et al., 2007 ; Fofana et al., 2017). Les opérations de désherbage sont particulièrement laborieuses et nécessitent entre 20 et 30 journées de travail par hectare (Froment \& Renard, 2001).

Cissé (1975) recommandait de procéder au premier sarclage un mois après semis, ce qui permet de distinguer plus facilement le fonio des graminées à grandes feuilles et des plants d'autres familles. Un second sarclage au début de la floraison est également favorable pour distinguer le fonio des autres graminées qui n'avaient pu l'être au premier sarclage. Par ailleurs, un désherbage entre 10 et 20 jours après semis a permis d'améliorer le rendement de $40 \%$ par rapport à un désherbage à plus de 30 jours (Vall et al., 2008). Ainsi, un désherbage tardif est à éviter en raison de la faible aptitude des jeunes plants de fonio à la compétition avec les adventices (Cruz et al., 2007). Il découle que le potentiel de croissance des variétés, la reconnaissance du fonio aux adventices semblables, la densité et le tallage sont autant de paramètres intéressants à prendre en compte pour mieux raisonner le contrôle des adventices. 


\section{MALADIES, RAVAGEURS DU FONIO ET MESURES DE LUTTE}

L'identification et la reconnaissance des maladies du fonio par la recherche a été progressive dans le temps.

Cissé a signalé, en 1975, la présence d'un ascomycète (Phyllachorasphaero sperma Winter) responsable de l'alternariose du fonio. Haq \& Ogbe (1995) ont souligné que le fonio est sensible à la rouille, maladie fongique causée par Puccinia cahuensis Henn. Vodouhè et al. (2003), à leur tour, ont signalé l'helminthosporiose et la cercosporiose sur les écotypes du fonio cultivés au Bénin.

L'ergot causé par le champignon Claviceps fusformis Loveless a aussi été rapporté comme l'une des principales affections paniculaires observées dans les champs des agriculteurs au Sénégal (Camara, 2011).

La lutte contre ces maladies est essentiellement préventive. Elle se fait par le choix des semences saines et résistantes, la rotation ou association culturale, l'arrachage et la destruction des plants malades pour éviter les infections ou infestations secondaires (Vodouhè et al., 2003 ; Cruz et al., 2007).

En outre, une étude sur la pénétration et le développement des juvéniles des nématodes Meloidogyne javanica Goldi et M. incognita Goldi par inoculation dans les racines du fonio a montré que le fonio est résistant à ces parasites et pourrait entrer en rotation avec les espèces potagères pour combattre ces derniers (Sarr \& Prot, 1985 ; Vodouhè \& Achigan Dako, 2006).

Par ailleurs, le fonio a toujours été considéré comme une culture très peu attaquée par les ravageurs. Des études entomologiques ont prouvé le contraire. Ainsi, les insectes ravageurs les plus connus sur le fonio sont les piqueurs-suceurs, les broyeurs, les foreurs et les coléoptères déprédateurs de stocks des grains paddy (Tableau 3). La figure 1 illustre les trois ravageurs déprédateurs des stocks de fonio les plus importants en milieu paysan (Ndao, 2011). Ces travaux montrent

Tableau 3. Principaux ravageurs du fonio et mesures de lutte - Main pests of fonio and control measures.

\begin{tabular}{|c|c|c|c|}
\hline Ravageurs & Dégâts & Mesures de lutte & Références \\
\hline Fourmis & $\begin{array}{l}\text { Transport des grains dans les } \\
\text { galeries }\end{array}$ & $\begin{array}{l}\text { Recouvrir immédiatement et } \\
\text { légèrement les grains après } \\
\text { semis }\end{array}$ & CRA Bareng, 2003 \\
\hline $\begin{array}{l}\text { Insectes piqueurs-suceurs } \\
\text { (punaises, pucerons, } \\
\text { acariens, jassides, } \\
\text { cantharides, etc.) }\end{array}$ & $\begin{array}{l}\text { Vidage des épillets à la phase } \\
\text { de fécondation }\end{array}$ & $\begin{array}{l}\text { Appliquer le mélange de } 80 \mathrm{ou} \\
100 \text { cc de sumithion à } 250 \mathrm{~g} \text { de } \\
\text { Topsin-M (thiophanate-méthyl) } \\
\text { dans } 10 \mathrm{~L} \text { d'eau }\end{array}$ & Camara, 2011 \\
\hline Insectes foreurs & $\begin{array}{l}\text { Creusage du cornet foliaire } \\
\text { et blocage du développement } \\
\text { des plants }\end{array}$ & $\begin{array}{l}\text { Appliquer le mélange de } 80 \text { ou } \\
100 \text { cc de sumithion à } 250 \mathrm{~g} \text { de } \\
\text { Topsin-M (thiophanate-méthyl) } \\
\text { dans } 10 \mathrm{~L} \text { d'eau }\end{array}$ & CRA Bareng, 2003 \\
\hline $\begin{array}{l}\text { Insectes broyeurs (larves } \\
\text { galéruques de coléoptères } \\
\text { et chenilles glabres à raies } \\
\text { longitudinales multicolores) }\end{array}$ & $\begin{array}{l}\text { Consommation du } \\
\text { parenchyme foliaire }\end{array}$ & $\begin{array}{l}\text { Appliquer le mélange de } 80 \mathrm{ou} \\
100 \text { cc de sumithion à } 250 \mathrm{~g} \text { de } \\
\text { Topsin-M (thiophanate-méthyl) } \\
\text { dans } 10 \mathrm{~L} \text { d'eau }\end{array}$ & Vodouhè et al., 2003 \\
\hline $\begin{array}{l}\text { Déprédateurs de stocks } \\
\text { (Tribolium confusum Val. } \\
\text { et } T \text {. Casteneum Herbst, } \\
\text { Rhyzopertha dominica } \\
\text { Fabricius, Ephestia cautella } \\
\text { Walker) }\end{array}$ & $\begin{array}{l}\text { Déprédation des grains } \\
\text { paddy du fonio stocké }\end{array}$ & $\begin{array}{l}\text { Appliquer le mélange de } 80 \text { ou } \\
100 \text { cc de sumithion à } 250 \mathrm{~g} \text { de } \\
\text { Topsin-M (thiophanate-méthyl) } \\
\text { dans } 10 \mathrm{~L} \text { d'eau }\end{array}$ & $\begin{array}{l}\text { Gueye \& Delobel, } 1999 \text {; } \\
\text { Ndao, } 2011\end{array}$ \\
\hline $\begin{array}{l}\text { Oiseaux granivores (pigeons, } \\
\text { tourterelles, passereaux, etc.) }\end{array}$ & $\begin{array}{l}\text { Attaque des meules de fonio } \\
\text { aux champs et dans les } \\
\text { greniers }\end{array}$ & $\begin{array}{l}\text { Surveillance durant les premiers } \\
\text { jours après le semis et juste } \\
\text { avant la récolte ; installation des } \\
\text { épouvantails }\end{array}$ & $\begin{array}{l}\text { Vodouhè et al., } 2003 \text {; } \\
\text { Cruz et al., } 2011\end{array}$ \\
\hline $\begin{array}{l}\text { Rongeurs (rats, aulacodes, } \\
\text { etc.) }\end{array}$ & $\begin{array}{l}\text { Attaque des meules de fonio } \\
\text { aux champs et dans les } \\
\text { greniers }\end{array}$ & $\begin{array}{l}\text { Destruction des niches de } \\
\text { rongeurs }\end{array}$ & $\begin{array}{l}\text { Vodouhè et al., } 2003 \text {; } \\
\text { Cruz et al., } 2011\end{array}$ \\
\hline $\begin{array}{l}\text { Autres animaux } \\
\text { (phacochères, singes, } \\
\text { animaux domestiques) }\end{array}$ & $\begin{array}{l}\text { Égrenage et consommation } \\
\text { des grains, déprédations dans } \\
\text { les champs de fonio }\end{array}$ & $\begin{array}{l}\text { Récolte à bonne date, } \\
\text { surveillance }\end{array}$ & $\begin{array}{l}\text { Vodouhè et al., } 2003 \text {; } \\
\text { Cruz et al., } 2011\end{array}$ \\
\hline
\end{tabular}




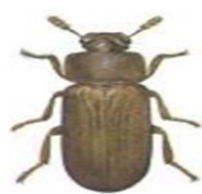

a. Tribolum casteneum

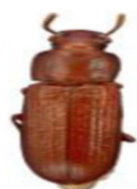

b. Tribolum confusum

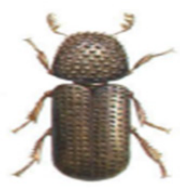

c. Rhyzopertha dominica
Figure 1. Principaux insectes coléoptères déprédateurs des stocks de fonio au Sénégal - Major Coleopteran insects pest of fonio stocks in Senegal.

Sources : Ndao, 2011 ; Gueye, 2016.

les avancées enregistrées en termes de connaissance de l'entomofaune nuisible, car Vodouhè \& Achigan Dako (2006) ont affirmé que les grains de fonio n'étaient pas sujets aux dégâts causés par les ravageurs déprédateurs des stocks et se conservaient bien. De même, des dégâts d'oiseaux granivores et de mammifères sont de plus en plus rapportés (Tableau 3). Les moyens de lutte utilisés sont variables selon les ravageurs et constitués généralement des techniques non chimiques (Tableau 3). Même si ce n'est pas courant, le recours aux insecticides chimiques a été mentionné (Vodouhè et al., 2003 ; Cruz et al., 2011). Des approches de lutte intégrée combinant des doses modérées d'insecticides et d'autres techniques (culturale, mécanique, biologique, etc.) pourraient être testées pour un meilleur contrôle de ces ravageurs.

\section{RÉCOLTE ET OBTENTION DU FONIO PADDY}

La récolte commence dès la maturité du grain, souvent avant que les pluies aient cessé (Cruz et al., 2007). Elle s'étend en général de septembre à novembre (USAID, 2008 ; Cruz et al., 2011) selon le type de cultivar, les besoins ou le calendrier cultural. A maturité, les feuilles jaunissent ou prennent la couleur violacée. Les grains brunissent et font tomber les tiges à la verse sous l'effet conjugué de leur poids et de la direction du vent (Fofana et al., 2017). Cruz et al. (2011) ont mentionné que la récolte se fait lorsque les pertes de grains par égrenage spontané deviennent inévitables. Les travaux récents de Gueye (2016) au Sénégal ont montré que la récolte doit intervenir une à deux semaines après la maturité physiologique. Elle peut être échelonnée pour répondre aux besoins immédiats du paysan (Vodouhè \& Achigan Dako, 2006). La récolte est demeurée manuelle et pénible. La technique consiste à récolter au couteau ou à la faucille et dans le sens de la verse naturelle. Cissé (1975) rapporte que les tiges sont fauchées sous la rosée à la faux pour les variétés qui se maintiennent dressées et à la faucille pour les variétés qui se couchent complètement sur le sol afin de réduire les pertes en grains (Figure 2). Le fauchage, très exigeant en main-d'œuvre, nécessite en général 20 à 30 hommes-jours par hectare (Cruz et al., 2011). La mécanisation de la récolte est difficile du fait de la verse des panicules et tiges (Vodouhè \& Achigan Dako, 2006). Leur relèvement est aussi difficile vu la finesse et la fragilité des tiges, favorisant ainsi l'égrenage spontané (Cruz et al., 2007 ; AdoukonouSagbadja, 2010 ; Cruz et al., 2011). Il n'existe pas encore de moissonneuse mécanique spécifique mise au point pour la récolte du fonio. Ceci constituerait un goulot d'étranglement à la production à grande échelle au regard du travail à consacrer à la récolte et les pertes par égrenage spontané qui pourraient aller jusqu'à $25 \%$ voire plus si la récolte est retardée (Vodouhè et al., 2003). Toutefois, le passage à la mécanisation de la récolte du fonio nécessiterait une étude sur la rentabilité et l'accessibilité de moissonneuses adaptées.

Le séchage commence au champ. Il se fait pendant plusieurs jours voire semaines afin de réduire la pourriture des grains (Vodouhè \& Achigan Dako, 2006 ; USAID, 2008).

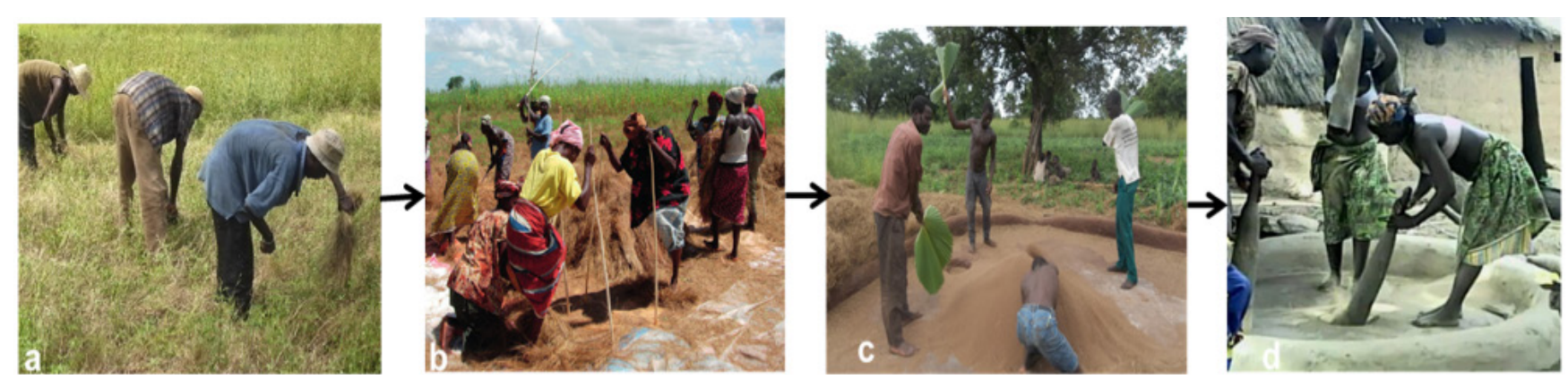

Figure 2. Succession artisanale des opérations de récolte et post-récolte du fonio - Traditionnal succession of harvesting and post-harvesting operations of fonio.

a. récolte manuelle à la faucille - manual harvest with sickle; b. battage par foulage - threshing by tramping ; $\mathbf{c}$. vannage manuel — manual winnowing; d. décorticage par pilage - dehulling by pounding. Sources : Vall et al., 2008 ; Cruz et al., 2011.

(C) Jean-François CRUZ, Cirad. 
Le battage se fait le plus souvent en groupe à l'aide de bâtons ou par piétinement (Figure 2). Il est souvent l'occasion de renforcement des liens sociaux à travers la liesse populaire que cela occasionne. Cependant, à l'instar de la récolte, le battage est manuel et pénible. Approximativement, par hectare, il requiert 15 à 20 hommes-jour (Cruz et al., 2011). L'usage d'une batteuse munie de secoueurs facilite le travail. C'est le cas de la batteuse ASSI d'un débit de $250-300 \mathrm{~kg} \cdot \mathrm{h}^{-1}$, adaptée à partir de la batteuse IRRI du riz (Cruz et al., 2011). La rentabilité du battage motorisé, la qualité du produit et l'accessibilité des batteuses conditionnent l'adoption et l'utilisation à grande échelle de ces matériels dans les zones de production du fonio.

Le vannage s'effectue à l'aide de paniers et calebasses en jouant sur la direction et le sens du vent. Après vannage, le fonio est séché au soleil pendant 8 à 10 jours jusqu'à $14 \%$ d'humidité ou en dessous (Cruz et al., 2007).

Les rendements du fonio paddy varient fortement en fonction de la variété, du sol, des conditions climatiques, du niveau d'entretien et d'infestation. Les rendements au champ sont souvent faibles, variant entre 200 à $900 \mathrm{~kg} \cdot \mathrm{ha}^{-1}$, mais peuvent atteindre voire dépasser $1000 \mathrm{~kg} \cdot h \mathrm{~h}^{-1}$, ou même redescendre à moins de $150 \mathrm{~kg} \cdot \mathrm{ha}^{-1}$ en régions marginales (Froment \& Renard, 2001 ; Vodouhè \& Achigan Dako, 2006 ; Cruz et al., 2011).

\section{DÉCORTICAGE ET CONSERVATION DU FONIO}

À l'instar de la récolte et du battage, le décorticage $\mathrm{du}$ fonio est longtemps resté manuel, très harassant et peu mécanisé. Il est consommateur de temps et de main-d'œuvre. En effet, réalisé habituellement par les femmes à l'aide de pilon et mortier, le décorticage demande trois à cinq pilages successifs séparés par des vannages. Elles ajoutent parfois du sable fin pour faciliter le décorticage par abrasion, ce qui nuit à la qualité du produit et nécessite d'importants criblages par plusieurs lavages pour éliminer le sable et les impuretés. Un ou deux pilages, vannages additionnels, permettent d'éliminer le son qui adhère encore au grain pour donner du fonio blanchi, la forme comestible. Le débit du décorticage et du blanchiment manuels demeure très faible, de l'ordre de $1-3 \mathrm{~kg} \cdot \mathrm{h}^{-1}$ et une femme ne peut dépasser 20 à $25 \mathrm{~kg}$ par jour (Sylla, 2005 ; Cruz et al., 2011). Le rendement au décorticage dépend du type variétal, du taux d'humidité et d'impuretés, du professionnalisme et du nombre de pilage-vannage. Il peut varier de 50 à $70 \%$.

Par ailleurs, plusieurs efforts de recherches ont été déployés essentiellement sur l'adaptation des décortiqueuses utilisées sur le riz, le mil ou le sorgho pour le décorticage mécanique du fonio (modèle Sanoussi [Sénégal], marque BCN [Mali] ; décortiqueuse GMBF de type Engelberg [Guinée, Mali, Burkina Faso et France], etc.) (Marouzé et al., 2008; Cruz et al., 2011). Ces décortiqueuses ne sont pas largement accessibles aux agriculteurs et malgré leurs capacités de décorticage supérieures à la technique manuelle (30-150 kg.h ${ }^{-1}$, voire plus), ne sont pas encore à la hauteur des attentes vu la taille minuscule des grains, l'adhérence des glumes, la qualité du produit issu du décorticage, etc. (Sylla, 2005 ; Cruz et al., 2011). L'affinement dans la mécanisation est nécessaire pour la mise au point de décortiqueuses adaptées et à grande puissance de production.

Le fonio est conservé sous forme de gerbes, de grains paddy, décortiqués ou blanchis dans des greniers, silos ou sacs en jutes et stockés dans des locaux secs et bien aérés. Les expériences paysannes au Nigeria ont montré que le fonio paddy, bien séché à 10-11\% d'humidité, peut être conservé pendant 25 ans (KwonNdung et al., 2001).

\section{CONCLUSIONS ET RECOMMANDATIONS}

Le fonio, céréale mineure, connaît aujourd'hui un regain d'intérêt au regard de ses potentiels et de sa position de culture biologique à valoriser pour la protection des écosystèmes et de l'environnement. Cependant, les techniques culturales situées en amont des chaines de valeur du fonio sont restées traditionnelles, très peu voire non améliorées malgré quelques efforts de recherche effectués dans les pays producteurs. Il en résulte des opérations culturales exigeantes en maind'œuvre et des rendements généralement faibles. Ainsi, aux plans national et régional, des projets de recherches innovants doivent être entrepris de manière concertée à chaque niveau de la chaine de production du fonio. La mécanisation et la recherche de pratiques de semis améliorées demeurent un défi en culture du fonio. Les tests de germination par le recours aux phytohormones devraient être étendus dans plusieurs zones pour réduire les difficultés de levée souvent observées. Des recherches sur le plan des fumures (formules, doses et dates d'apport) doivent être affinées pour répondre aux exigences agroécologiques de chaque zone. D'autres aspects à explorer seraient le recours aux biofertilisants et les associations avec les légumineuses de couverture. Les essais de lutte biologique contre les ravageurs, notamment l'utilisation d'extraits botaniques, devraient être aussi testés. En prélude à une lutte efficace, un inventaire holistique des nuisibles et leurs dégâts dans les aires de culture du fonio s'avère nécessaire. La mécanisation de la récolte ou l'adaptation de moissonneuses utilisées sur les autres céréales, comme le riz, devraient être explorées. La mécanisation des 
autres activités post-récolte en cours devrait être affinée et largement étendue. La rentabilité et l'accessibilité de ces divers progrès techniques et l'écoulement facile des produits sur le marché permettraient l'adoption des nouvelles pratiques élaborées et d'insuffler une relance effective à la production du fonio.

\section{Bibliographie}

Adoukonou-Sagbadja H., Dansi A., Vodouhè R. \& Akpagana K., 2004. Collecting fonio (Digitaria exilis Kipp. Stapf, D. iburua Stapf) landraces in Togo. Plant Genet. Resour. Newsl., 139, 63-67.

Adoukonou-Sagbadja H., Dansi A., Vodouhè R. \& Akpagana K., 2006. Indigenous knowledge and traditional conservation of fonio millet (Digitaria exilis, Digitaria iburua) in Togo. Biodivers. Conserv., 15(8), 2379-2395, doi.org/10.1007/s10531-004-2938-3

Adoukonou-Sagbadja H., 2010. Genetic characterization of traditional fonio millets (Digitaria exilis, D. iburua STAPF) landraces from West-Africa: implications for conservation and breeding. $\mathrm{PhD}$ thesis: Justus-Liebig University Giessen (Germany).

Aliero A. \& Morakinyo A., 2005. Photoperiodism in Digitaria exilis Stapf accessions. Afr. J. Biotechnol., 4(3), 241-243.

Bakare O., 2005. Influence of time of harvest on grain yield of acha, Digitaria exilis and farmers' perspectives. Int. J. Agric. Rural Dev., 6, 132-135, doi.org/10.4314/ijard. v6i1.2599

Barnaud A. \& Billot C., 2011. Compte rendu de conférence. Atelier international "De la connaissance à la valorisation du fonio" 2010, organisé par le Cirad, l'IRD, l'université Abdou Moumouni de Niamey, l'IRAG et le projet ARCAD, Niamey, Niger, 9-11 décembre 2010. Cah. Agric., 20(4), 310-312.

Camara M., 2011. Détermination du focus parasitaire du fonio (Digitaria exilis Stapf) en Casamance continentale et au Sénégal oriental. Mémoire de DEA: École Nationale Supérieure d'Agriculture de Thiès (Sénégal).

CIRAD, 2009. La plante du fonio, https://fonio.cirad.fr/, $(29 / 06 / 2020)$.

Cissé I.B., 1975. La culture de fonio et quelques aspects écophysiologiques de la plante. Wageningen, The Netherlands : Landbouwhogeschool.

CRA Bareng, 2003. Fiche technique-Fonio en Guinée. In : Vodouhè S.R., Zannou A. \& Achigan Dako E., éds. Actes $d u$ Premier atelier sur la diversité génétique $d u$ fonio (Digitaria exilis ) en Afrique de l'Ouest, 4-6 aout 1998, Conakry, Guinée. Rome : Institut International des Ressources Phytogénétiques (IPGRI), 68-69.

Cruz J.-F., Dupuis B., Forest F. \& Stilmant D., 2007. D32. Étude bibliographique: inventaire des connaissances en termes de variétés cultivées en Guinée, au Mali et au Burkina Faso et inventaire des connaissances en termes de systèmes de culture incluant la production de fonio dans ces trois pays : projet $n^{\circ} 015403$ FONIO. Amélioration de la qualité et de la compétitivité de la filière fonio en Afrique de l'Ouest. Montpellier, France : Cirad.

Cruz J.F., Béavogui F. \& Dramé D., 2011. Le fonio, une céréale africaine. Versailles, France: Éditions Quæ; Wagneningen, Pays-Bas: CTA; Gembloux, Belgique: Presses agronomiques de Gembloux, doi. org/10.35690/978-2-7592-1040-4

Dachi S.N., Mamza W.S. \& Bakare S.O., 2017. Growth and yield of acha (Digitaria exilis Kippis Stapf) as influenced by sowing methods and nitrogen rates in the Guinea savanna area of Nigeria. FULafia J. Sci. Technol., 3(2), 33-37.

Dansi A., Adoukonou-Sagbadja H. \& Vodouhe R., 2010. Diversity, conservation and related wild species of fonio millet (Digitaria spp.) in the northwest of Benin. Genet . Resour. Crop Evol., 57(6), 827-839, doi.org/10.1007/ s10722-009-9522-3

Diallo T.A., Fliedel G., Stilmant D. \& Cruz J.-F., 2008. Catalogue de quelques écotypes de fonio de Guinée, du Mali et du Burkina Faso. Projet Fonio. In : Cruz J.F., ed. Amélioration de la qualité et de la compétitivité de la filière fonio en Afrique de l'Ouest [CD-Rom]. Montpellier, France : Cirad.

Fall C.A., Fofana A. \& Baldé S., 2005. Recherche agronomique sur le fonio (Digitaria exilis Stapf.) au Sénégal: diagnostic des systèmes de production et sélection de variétés performantes. Dakar: ISRA.

Fofana A. et al., 2017. Fiche technique du fonio. Sér. Fiche Techn. ISRA, 14(4), 11.

Froment D.\&Renard C., 2001.Fonio.In :Raemaekers R.H., ed. Agriculture en Afrique tropicale. Bruxelles: DGCI, 37-43.

Gigou J. et al., 2009. Fonio millet (Digitaria exilis) response to $\mathrm{N}, \mathrm{P}$ and $\mathrm{K}$ fertilizers under varying climatic conditions in West Africa. Exp. Agric., 45(4), 401-415, doi.org/10.1017/s0014479709990421

Gueye M., 2016. Amélioration des techniques de semis, de fertilisation et de récolte du fonio blanc (Digitaria exilis Stapf ; Poaceae) au Sénégal. Thèse de doctorat: Université Cheikh Anta Diop de Dakar (Sénégal).

Gueye M.T. \& Delobel A., 1999. Relative susceptibility of stored pearl millet products and fonio to insect infestation. J. Stored Prod. Res., 35, 277-283, doi. org/10.1016/s0022-474x(99)00011-9

Gueye O., 2007. Typologie et caractérisation des micro et petites entreprises de transformation du fonio en Afrique de l'Ouest (Guinée, Mali, Sénégal). Enda Graf, IER, IRAG, Projet FONIO/UE, Delivrable 1.

Haq N. \& Ogbe D.F., 1995. Fonio (Digitaria exilis and D.iburua). In: Williams J.T., ed. Cereals and pseudocereals. London: Chapman and Hall, 225-245.

Idu M., Chokor J.U. \& Timothy O., 2008. Effect of various hormones on the germination of fonio Digitaria exilis L. Int.J.Bot., 4, 456-460, doi.org/10.3923/ijb.2008.456.460 
Jideani I.A., 1990. Acha, Digitaria exilis, the neglected cereal. Agric. Int. (UK), 42(5), 132-143.

Kanlindogbe C. et al., 2018. Évaluation participative de types et écartements de semis en culture de fonio dans la commune de Boukombé, nord-ouest du Bénin. In : Direction de la Recherche et de l'Innovation, éd. $18 \grave{e}$ édition des Journées Scientifiques Internationales de Lomé, 8-13 octobre 2018, Lomé, Togo.

Kwon-Ndung E.H., Misari S.M. \& Dachi S.N., 2001. Study on the production practices of acha (Digitaria exilis Kippis Stapf.) in Nigeria. Sci. Forum, 4(2), 106-113.

Marouzé C. et al., 2008. Designing a fonio mill, screening an operating principle and its validation. Agric. Mechanization Asia Africa Latin America, 39, 9-15.

Ndao A., 2011. Diversité, abondance et prévalence des principaux coléoptères ravageurs de stocks de fonio, de maïs et de mil au Sénégal. Mémoire de Master II : Faculté des Sciences et Techniques, Université Cheikh Anta Diop, Dakar (Sénégal).

Ndiaye M., Termorshuizen A.J. \& van Bruggen A.H., 2008. Effect of rotation of cowpea (Vigna unguiculata) with fonio (Digitaria exilis) and millet (Pennisetum glaucum) on Macrophomina phaseolina densities and cowpea yield. Afr. J. Agric. Res., 3(1), 037-043.

Ndor E., Mamman B.D., Jayeoba O.J. \& Dauda S.N., 2016. Effect of organic and inorganic fertilizers on yield and economic return of acha (Digitaria spp.) varieties in Lafia, Nigeria. Global J. Agric. Res., 4(3), 13-20.

Ndoye F. et al., 2016. Réponse du fonio blanc (Digitaria exilis Stapf) à l'inoculation avec des champignons mycorhiziens à arbuscules en conditions semi-contrôlées . J. Appl. Biosci., 103(1), 9784-9799.

Ouedraogo I., Hema O.S. \& Sanou A., 2015. Inventaire et incidence des insectes inféodés à la culture du fonio (Digitaria exilis Stapf) dans la zone ouest du Burkina Faso. J. Appl. Biosci., 94, 8880-8889, doi.org/10.4314/ jab.v94i1.11

Philip T. \& Itodo I., 2006. Acha (Digitaria spp.) a "rediscovered" indigenous crop of West Africa. Agric. Eng. Int. CIGR Ejournal Invited Overview, 8(23).

Portères R., 1955. Les céréales mineures du genre Digitaria en Afrique et en Europe. J. Agric. Trop. Bot. Appl., 2(79), 349-386.

Portères R., 1976. African cereals: eleusine, fonio, black fonio, teff, Brachiaria, paspalum, Pennisetum, and African rice. In: Harlan J.R., de Wet J.M.J. \& Stemler A.B.L., eds. Origins of African plant domestication. The Hague: Mouton Publishers, 409-452.
Renoux L.\& Dumas P., 1905. Culture du fonio dans la vallée du Sénégal et du Haut Niger. L'agriculture pratique des pays chauds. Bull. Jard. Col. Nogent, 32, 357-359.

Sarr E. \& Prot J.C., 1985. Pénétration et développement des juvéniles d'une souche de Meloidogyne javanica et d'une race B de $M$. incognita dans les racines du fonio (Digitaria exilis Stapf). Rev. Nématol., 8(1), 59-65.

Sekloka E. et al., 2015.Évolution de la diversité des cultivars de fonio pratiqués à Boukoumbé et environs. Int. J. Biol . Chem. Sci., 9(5), 2446-2458, doi.org/10.4314/ijbcs. v9i5.16

Somda B.B. et al., 2017. Détermination des doses optimales de fumures organo-minérales en microdose dans la zone soudano-sahélienne du Burkina Faso. Int. J. Biol. Chem. Sci., 11(2), 670-683.

Sylla, 2005. Capitalisation d'informations sur la filière fonio au Mali. Montreuil, France : Afrique Verte.

Traore D. et al., 2009. Food forms and beta glucans contents in sorghum, fonio and rice can influence their glycemic indexes. FASEB J., 23(1), Suppl. 563-28.

USAID, 2008. Chaîne de valeurs de la filière fonio au Sénégal. Washington DC, USA: International Resources Group.

Vall É., Dembélé K., Kanwé A. \& Cruz J.F., 2008. D28. Options pour le développement de la production de fonio, l'amélioration de la productivité et de la compétitivité : projet $n^{\circ} 015403$ FONIO. Amélioration de la qualité et de la compétitivité de la filière fonio en Afrique de l'Ouest. Bobo-Dioulasso, Burkina Faso : CIRDES.

Vall É., Andrieu N., Beavogui F. \& Sogodogo D., 2011. Les cultures de soudure comme stratégie de lutte contre l'insécurité alimentaire saisonnière en Afrique de l'Ouest: le cas du fonio (Digitaria exilis Stapf). Cah. Agric., 20(4), 294-300, doi.org/10.1684/agr.2011.0499

Vodouhè S.R., Zannou A. \& Achigan Dako E.G., 2003. Actes du Premier Atelier sur la Diversité Génétique du Fonio (Digitaria exilis) en Afrique de l'Ouest, Conakry, Guinée, du 4 au 6 aout 1998. Rome : Institut International des Ressources Phytogénétiques (IPGRI).

Vodouhè S.R. \& Achigan Dako E.G., 2006. Digitaria exilis (Kippist) Stapf. In: Brink M. \& Belay G., eds. PROTA (Plant Resources of Tropical Africa/ Ressources végétales de l'Afrique tropicale), Wageningen, Pays-Bas, http://uses.plantnetproject.org/f/index. php?title=Digitaria_exilis_(PROTA)\&oldid=95268, (9/07/2018).

(47 réf.) 